ISSN: 2578-3335 (Print) 2578-3343 (Online)

Volume 2 | Issue 1

Article 7

2020

\title{
Acupuncture and its Effects on Chronic Pain
}

Pavan Patel

Mercy Health System, pavan.patel@mercyhealth.org

Michael Sabia

Cooper Medical School of Rowan University, sabia-michael@cooperhealth.edu

Jashen Patheja

Drexel University, jpatheja@gmail.com

Rohan Kapoor

Georgetown University, rohankapoor48@gmail.com

Tiffany Mathias

Drexel University, tiffanymathias@gmail.com

See next page for additional authors

Cooper Rowan Medical Journal: https://rdw.rowan.edu/crjcsm

Would you like to be a reviewer? Please fill in this short form to express your interest.

\section{Recommended Citation}

Patel, Pavan; Sabia, Michael; Patheja, Jashen; Kapoor, Rohan; Mathias, Tiffany; and Murtaza, Tameem (2020) "Acupuncture and its Effects on Chronic Pain," Cooper Rowan Medical Journal: Vol. 2 : Iss. 1 , Article 7.

DOI: 10.31986/issn.2578-3343_vol2iss1.7

Available at: https://rdw.rowan.edu/crjcsm/vol2/iss1/7

\section{(c) (7)}

This work is licensed under a Creative Commons Attribution 4.0 License.

This Reviews is brought to you for free and open access by the Rowan University Journals at Rowan Digital Works. It has been accepted for inclusion in Cooper Rowan Medical Journal by an authorized editor of Rowan Digital Works. For more information, please contact brush@rowan.edu. 


\section{Acupuncture and its Effects on Chronic Pain}

\section{Authors}

Pavan Patel, Michael Sabia, Jashen Patheja, Rohan Kapoor, Tiffany Mathias, and Tameem Murtaza 


\title{
Acupuncture and its Effects on Chronic Pain
}

\author{
Pavan Patel, D.O. ${ }^{1 *}$, Michael Sabia, M.D. ${ }^{2}$, Jashen Patheja, M.D. ${ }^{3}$, Rohan Kapoor, \\ M.D. ${ }^{4}$, Tiffany Mathias, M.D. ${ }^{5} \&$ Tameem Murtaza, M.D. ${ }^{6}$ \\ ${ }^{1}$ Neurology Resident, Einstein Medical Center, Philadelphia, PA, United States \\ ${ }^{2}$ Anesthesiology, Cooper Medical School of Rowan University, Camden, NJ, United States \\ ${ }^{3}$ Anesthesia Fellow, New York Medical College, Valhalla, NY, United States \\ ${ }^{4}$ Pain Medicine Fellow, Wayne State University, Detroit, MI \\ ${ }^{5}$ Pain Medicine Fellow, Stanford University, Stanford, CA \\ ${ }^{6}$ Family Practice Resident, St. Joseph University, Philadelphia, PA \\ *Corresponding author: pavan.patel@mercyhealth.org (Pavan Patel, D.O.)
}

\begin{abstract}
Acupuncture is a complementary form of medicine that has relatively recently become a component of traditional Chinese medicine (TCM). It involves the application of thin needles at specific points throughout the body, although initially, general areas instead of points were used. There are estimated to be about 395 different points that can be utilized, and the technique behind using these points can vary widely between practitioners. In addition to acupuncture points, TCM is said to use meridians or pathways along with qi, or energy flows. In terms of acupunctures' utility, it has been known to treat a variety of ailments ranging from pain and headaches to sleep disturbances, but it's true benefits continue to be questioned. To date, thousands of studies have been performed in regards to its efficacy with varying conclusions. Once considered to be "pseudomedicine", this ancient technique has been found to provide long-term benefits for patients with chronic pain. This manuscript aims at providing a brief history of acupuncture and examines studies in favor of its relief of chronic pain.
\end{abstract}

Keywords: Chronic Pain, Acupuncture, Meridians, Opioid Receptors, NMDA, Low Back Pain, Sciatica, Myofascial Pain, Spinal Stenosis, Fatigue, Post Surgical Pain, Pain

\section{INTRODUCTION}

Acupuncture technique and practice was first observed in a two-thousand year old text called the Huangdi Neijing (Yellow Emperor's Classic on Internal Medicine). ${ }^{1}$ Its ideology takes root in the concept of duality, represented by two opposing, yet complementary forces called yin and yang. According to ancient 
Chinese philosophy, these two forces act throughout the entire cosmos. An imbalance of the two forces within the body precipitates physical disease or disharmony. When this imbalance occurs, the natural flow of qi, or life force, is hindered. The goal of acupuncture is to restore the normal distribution of yin and yang allowing the qi to once again flow unimpeded. ${ }^{2}$

Throughout the centuries, acupuncture gradually became a staple of Chinese therapy alongside diet, massage, moxibustion (heat) and herbs. The art of acupuncture disseminated throughout Korea and Japan in the sixth century, and eventually to Europe in the sixteenth century through reports by Jesuit missionaries. In 1971, acupuncture made its debut in the United States when a US press officer received the therapy after undergoing an emergency appendectomy in China. ${ }^{3}$ After writing about his experience in The New York Times, American physicians began to explore the utility of acupuncture in surgical pain control. In 1972 acupuncture analgesia was first utilized in the US operating room for an inguinal hernia repair and skin-graft operation, yielding mixed results. ${ }^{4}$

Over time the potential value of acupuncture was observed in myofascial pain syndromes and trigger points, encouraging further research to be conducted in the field. Following this, the National Institute of Health acknowledged the utility of acupuncture for pain relief in 1997, allowing it to enter the conventional medicine sphere. Acupuncture continues to grow today with multiple applications in the realm of pain medicine.

\section{METHODS}

Researching the effectiveness of acupuncture is challenging. While many studies have been performed, the quality of each of those studies varies greatly. In addition, many trials used "sham acupuncture" to compare against "real acupuncture" but the clinical application and quality of these procedures can lead to false negatives in terms of acupunctures true efficacy making it even more difficult to sift through the numerous studies.

The database used for the "Current Uses of Acupuncture" was Google Scholar. Search terms included acupuncture in conjunction with: headache, low back pain, sciatica, myofascial pain, stress, insomnia, tendonitis, spinal stenosis, osteoarthritis, and post-surgical pain. The inclusion criteria involved acupuncture's use for pain (low back pain, myofascial pain, chronic pain, etc). Exclusion criteria were acupunture's effects on Fertility, Nausea, and sleep.

\section{Meridians}

In traditional Chinese medicine, the jing lou, or meridian, is the non-physical pathway that qi flows through within the body. Each organ possesses its own meridian, and it is via specific acupoints located along these pathways that qi can be influenced. ${ }^{5}$ According to clinical observations that have existed for 
thousands of years, it is believed that there are 12 main meridians and 8 secondary meridians that run head to toe, and about 395 acupoints that connect them. ${ }^{6}$ With regard to location, the principal meridians are oriented bilaterally and symmetrically, however there are also midline meridians and intersecting meridians that connect the different channels. ${ }^{7}$

Multiple studies have focused on deducing the functional basis of meridians. Within the acupuncture community, there has been no consistent method to identify the location of meridians and acupoints, suggesting that these two entities may not exist in a physical basis. One interpretation of acupoints and meridians involves trigger points and their ability to be treated with acupuncture. Given that trigger points often orient in radicular patterns, these tender spots may be interpreted as acupoints and the patterns they manifest as the meridian. The location of meridians and acupoints often coincides with major neural pathways, such as the deep radial nerve and median nerve for example. The close proximity between these nerve structures and acupoints further reinforces the theory that meridians may be neuronally modulated. ${ }^{7}$ The study of acupoints and understanding of its mechanisms are continually evolving. Having been first conceptualized thousands of years ago as channels for the qi, meridians and the acupoints they contain have been studied extensively. While research has provided multiple prudent explanations for meridians, there still remains much to learn about their mechanism of action and clinical implications.

Figure 1 "Human Body Meridians" on the next page: 


\section{Human body meridians}
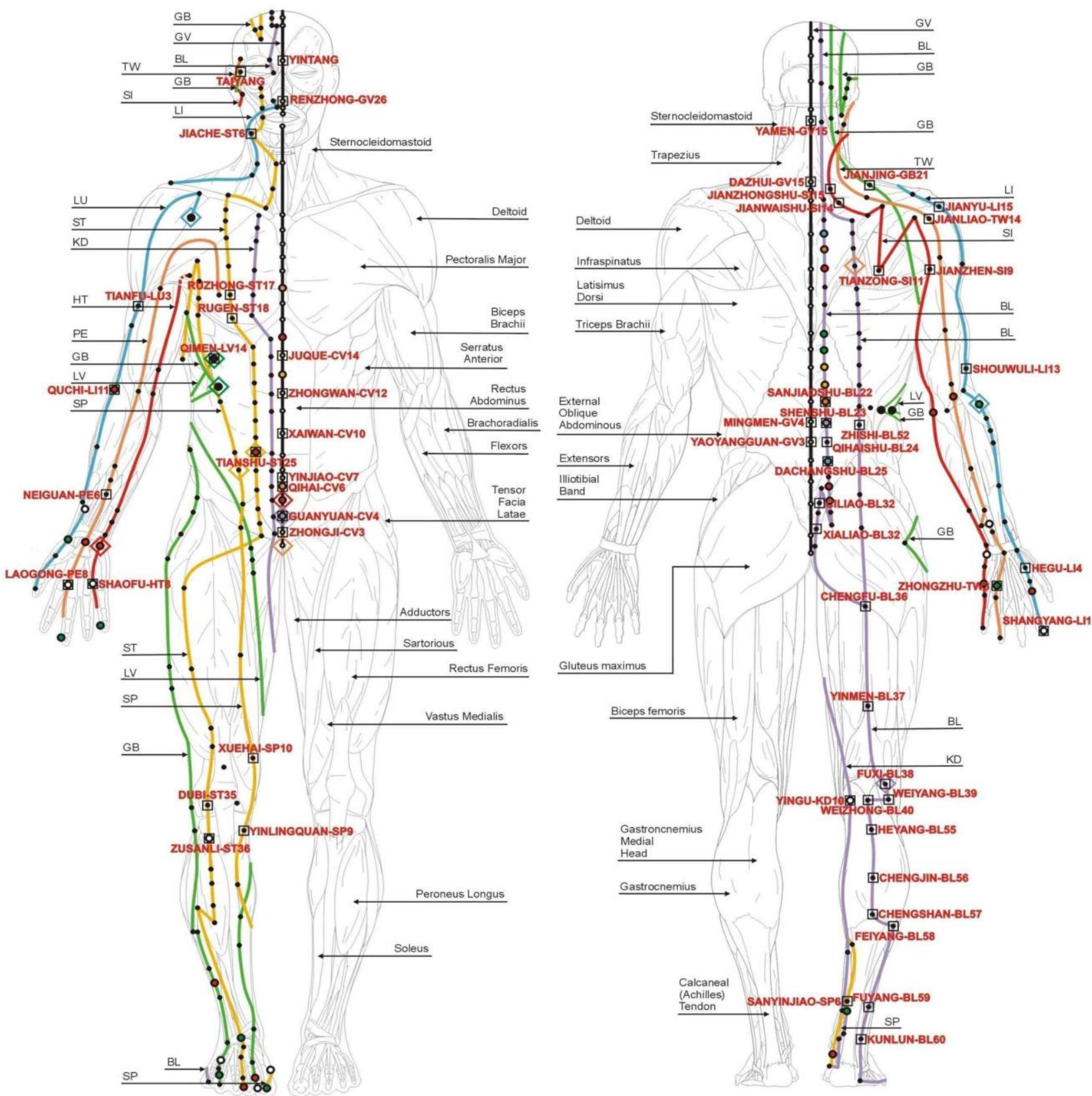

\section{ANTERIOR VIEW}

LEFT - YIN SUPERFICIAL MERIDIANS

ARM YIN MERIDIANS \& SHICHEN LEG YIN MERIDIANS \& SHICHEN

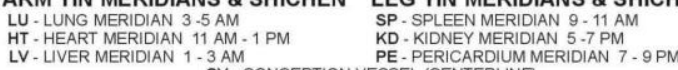
CV.CONCEPTION VESSEL (CENTERLINE

\section{POSTERIOR VIEW}

LEFT - SUPERFICIAL MUSCULATURE

RIGHT - YANG SUPERFICIAL MERIDIANS

ARM YANG MERIDIANS \& SHICHEN LEG YANG MERIDIANS \& SHICHEN

$\begin{array}{ll}\text { LI. LARGE INTESTINE MERIDIAN 5.7 AM } & \text { ST. STOMACH MERIDIAN 7.9 AM } \\ \text { SI. SMALL INTESTINE 1.3PM } & \text { BL- BLADDER MERIDIAN 3.5 PM }\end{array}$

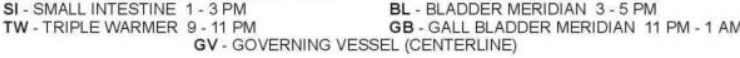
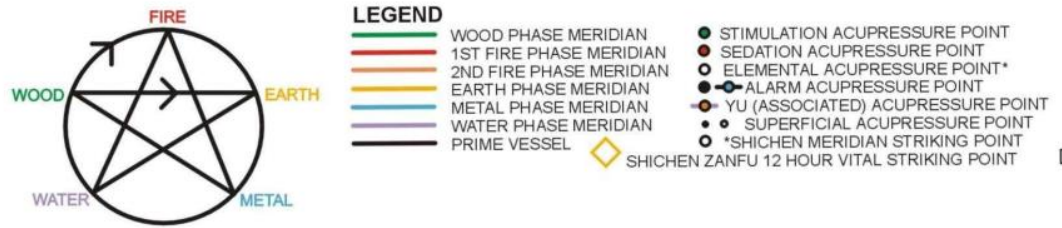

WRIST PULSE

LEFT

HT $/ L I$
LV $/ G B$

$\mathrm{KD} / \mathrm{BL}$

RIGET / SUPERFICIAL

DEEP/S
LU/L
SP/ST
$\mathrm{KD} / \mathrm{PE}$.

SP/ST
KD / PE - TW

$\square$ GENERAL USE STRIKING POINTS

Figure 1 A schematic of the "chinese" or human body meridians. The schematic was based on an image of the

Northern Crane Martial Arts Association dojo. This image is in the public domain and is hosted at: https://commons.

wikimedia.org/wiki/File:Chinese_meridians.JPG 


\section{Mechanisms of pain relief in acupuncture}

The mechanisms by which acupuncture facilitates pain relief is a topic that has been researched for some time with somewhat nebulous and a lack of unified results. While some studies hypothesize that acupuncture causes microinjury, 8 thereby changing the balance of serotonin and dopamine levels modulated within a localized area, others point to increased local blood flow and expedited healing processes.9,10 Another hypothesis is that acupuncture may trigger a somatic autonomic reflex that modulates gastric and cardiovascular functions.11 Opioid receptors are the most well studied factor that is manipulated in acupuncture.

When a needle is placed in the proper position, it induces the local release of opioids, which then activate peripheral nerve terminal receptors to suppress nociception. This in turn triggers the

hypothalamus-pituitary axis to release COX-2, which interferes with endocannibanoid metabolism. In turn, this leads to higher levels of opioids at the needle site.12 These opioids then act on peripheral nerve receptors to suppress nociception.13,14

At the peripheral level, it is widely acknowledged that opioid receptors play a role in pain modulation. Beta endorphins and met-enkaphalins also modulate pain. Within the spine, serotonin and norepinephrine, as well as glial cells, manipulate cytokine levels and subsequently modulate glutamate levels.

Administration of Naloxone or Naltrexone has consistently reversed the analgesic effects in a wide variety of patients who report pain relief with acupuncture. This reinforces the endogenous agonistic analgesic opioid effects/properties acupuncture exhibits. In a recent study in rats, electroacupuncture significantly decreased TNF-a, IL-1B and IL-6 expression in inflamed skin. ${ }^{15}$ Other studies have examined corticotropin releasing factor (CRF) and Prostaglandin E-2 in relation to acupuncture. They found that electroacupuncture may induce skin fibroblasts to release CRF, another opioid release modulator known to release opioids from inflammatory cells. ${ }^{16}$

At the spinal level, increased serotonin and norepinephrine release during acupuncture has been well documented in laboratory studies in rats, but without detailed understanding of their subsequent effect at the next level. ${ }^{17} \mathrm{~A}$ recent study showed promising results of a significant dosage decrease of SSRIs prescribed to patients who took paroxetine and also underwent acupuncture in comparison to those patients who received paroxetine alone. ${ }^{18}$ Interestingly, glial cells and microglia are well studied in this realm. Acupuncture is known to cause the inhibition of glial cell activation, which subsequently decreases cytokine release and increases pain alleviation. ${ }^{19}$ Substance $\mathrm{P}$ is also involved through the transmission of noxious inputs and the further activation of glial cells. Electroacupuncture increases the activity of mu and delta opioid receptors, thereby blocking substance $\mathrm{P}$ release ${ }^{20}$ Microglia also play an active role by inhibiting extracellular signaling molecules such as P38 and MAPK, causing a downstream decrease in 
molecules such as IL-1B, TNF-a, nitric oxide synthase, and prostaglandin E-2 in the lumbar spinal cord. ${ }^{21}$ The mechanism of pain relief at the supraspinal level is significantly less studied. It is known that the cortex, the thalamus, and several nuclei each play a role in modulating the N-methyl-D-aspartate receptor, which in turn changes the dimension of pain felt. ${ }^{22}$ Electroacupuncture is known to cause higher activation at the right insula, pulvinar, and medial nucleus of the thalamus when performed, particularly in patients with irritable bowel syndrome. ${ }^{23}$

Above the spinal cord, the thalamus and cerebral cortex also play a role in pain and temperature modulation. Some studies have found that electroacupuncture in particular heightens modulation of many of these aspects.

Who can do acupuncture?

Acupuncture is known for its diversity in terms of application, but it is also diverse in terms of the individuals that can perform it. Many providers from a variety of medical backgrounds can perform it. Of course, there are licensed acupuncturists, but beyond those individuals, chiropractors, physicians (both MD and DO), naturopaths, nurse practitioners, physical therapists and physicians assistants can all perform acupuncture, but requirements acorss states vary widely. More and more medical professionals are bringing acupuncture into their daily practice and with that, the regulations on training and administration are constantly being updated in order to ensure that patients receive safe, up-to-date and effective care. For example, in New Jersey, physicians, surgeons and dentists may practice acupuncture provided their coursework or training included acupuncture. In addition, a physician, either MD or DO, may practice acupuncture after completing 300 hours of training, including 150 hours of clinical training. In fact, as of 2018, there were over 900 licensed acupuncturists in New Jersey from a variety of backgrounds as noted before. However, in this state, podiatrists, physician assistants or chiropractors are not allowed to practice acupuncture. Guidelines for each state continue to evolve and further studies should analyze the outcomes of acupuncture treatment in order to help delineate future guidelines to ensure adequate and effective training. ${ }^{24}$

Table 1 "Mechanisms of Pain relief in Acupuncture Summary" on the next page: 
Table 1 Mechanisms of Pain relief in Acupuncture Summary

\begin{tabular}{|c|c|c|}
\hline Location & Component & Effect \\
\hline Supraspinal & Cortex, Thalamus and Nuclei & $\begin{array}{l}\text { - Modulation of N-methyl D As- } \\
\text { partate receptor } \\
\text { - Pain and temperature modula- } \\
\text { tion }\end{array}$ \\
\hline Spinal & Spinal cord neurons & $\begin{array}{l}\text { - Increased serotonin and NE re- } \\
\text { lease } \\
\text { - Inhibition of glial cell activation }\end{array}$ \\
\hline & Onioid rocontorc & $\begin{array}{l}\text { - Increased activity of mu and } \\
\text { delta opioid receptors } \\
\text {-Inhibition of extracellular sig- } \\
\text { naling molecules downstream de- } \\
\text { crease in inflammatory molecules } \\
\text { such as IL-1B, TNF-a, NO syn- }\end{array}$ \\
\hline Peripheral & $\begin{array}{l}\text { Opioid receptors, } \\
\text { Beta-endorphins, meta- } \\
\text { enkephalins }\end{array}$ & $\begin{array}{l}\text { thase, PGE2 } \\
\text { - Modulation of pain through de- } \\
\text { creased expression of TNF-a, IL- } \\
\text { 1B, IL-6 in inflamed skin } \\
\text { - Release of CRF release of opi- } \\
\text { oids from inflammatory cells }\end{array}$ \\
\hline
\end{tabular}




\section{Current Uses of Acupuncture}

Headaches are a significant source of health, economic and social costs on a daily basis. Even with the use of multiple medications, many patients continue to suffer from headaches without any relief. Of all the non-pharmacological approaches to managing headaches, acupuncture seems to be the most popular and seems to have significant benefits. A recent randomized, controlled trial with 401 patients suffering from chronic headaches, predominantly migraines revealed persistent and clinically relevant benefits. The primary outcome measured was the patients headache score at 1 year. Headache score at 12 months was lower in the acupuncture group $(16.2, \mathrm{SD} 13.7, \mathrm{n}=161,34 \%$ reduction from baseline) than in controls $(22.3, \mathrm{SD} 17.0, \mathrm{n}=140,16 \%$ reduction from baseline $) .{ }^{25}$

Low back pain is a common, impairing and disabling condition, often long-term, with an estimated lifetime prevalence of $70 \%$ to $85 \%$. It is the most common cause of job-related disability and a leading contributor to missed work days. A variety of treatment options are available to patients including physical therapy, over-the-counter and prescription medications, strengthening exercises, and steroid injections, amongst many more. Acupuncture has shown to be moderately effective in treating low back pain. A patient and observer randomized, controlled trial involving almost 1200 patients with chronic low back pain studied verum (traditional) and sham acupuncture versus conventional therapy consisting of the modalities listed previously. It found that low back pain improved after acupuncture treatment for at least 6 months. In addition, it found that treatment with either verum or sham acupuncture resulted in improvement almost twice that of conventional therapy. ${ }^{26}$ A recent systematic review of acupuncture and low back pain showed improved pain and symptomatology when comparing acupuncture to non-steroidal anti-inflammatory drugs and other drugs as well as sham procedures, but did not improve functionality or disability. The evidence is encouraging that acupuncture can help with symptom improvement and pain relief, but further studies should continue to be conducted in order to help solidifiy thesefindings. 27 Fibromyalgia is characterized by widespread pain, fatigue, and sleep disturbances. In addition, the combination of these factors contribute to patients suffering from somatic and psychological symptoms. The cause of this condition is unknown, but it is thought to be due to genetic and environmental factors. Because the pathogenesis of the condition is not well understood, there is no universal treatment or cure. Treatment typically consists of symptomatic management and trial and error. Acupuncture has and continues to be used to treat patients with this condition, but studies on its effectiveness have been mixed in terms of results. A recent systematic review analyzed results of acupuncture for patients diagnosed with fibromyalgia and a variety of outcomes such as pain, physical function, fatigue, sleep, total well-being, stiffness and adverse events. Ultimately, they found low to moderate evidence that compared to no treatment and standard therapy, acupuncture improves pain and stiffness. However, there was moderate 
evidence that acupuncture compared to sham does not work in reducing pain or fatigue, or improving sleep or global well-being. Overall, there appears to be some benefit, but larger studies are recommended for a more thorough and evidence based clinical picture. ${ }^{28}$

Sciatica is a condition that involves pain going down the leg from the lower back. Frequently, it is due to a spinal disc herniation, which subsequently presses on nerve roots. However, there are other causes including spinal stenosis, piriformis syndrome or spondylolisthesis, to name a few. There aren't many studies on the use of acupuncture and sciatica, but they are starting to increase in number. A systematic review and meta-analysis of eight different databases involving patients treated with acupuncture for this condition revealed that it may be effective in treating pain. 29

Myofascial pain is characterized by chronic pain in multiple points throughout the body. These points are known as myofascial trigger points and can present as focal points of tenderness with reproduction of the pain upon palpation. The cause of this condition is not known and there have been some studies refuting the idea of trigger points. Treatment of myofascial pain typically involves stretching and range of motion exercises. Small, low-quality studies have demonstrated benefits with dry needling, but these results are not very reliable. Ten randomized, controlled trials, comparing acupuncture and sham or no intervention, were studied using a meta-analysis. This revealed that acupuncture may be beneficial in terms of pain relief and reduced muscle irritability. 30

Stress is a type of psychological pain that can have significant negative health effects on the body. There are theories that acute and chronic stress have a relationship with illness. It can also be related to the development of depression and anxiety. A small study on acupunctures ability to attenuate stress showed a significant reduction in depression, anxiety and stress. ${ }^{31}$ Further studies are needed to analyze the relationship between acupuncture and stress, but as mentioned previously, it has shown some promising effects. In addition to general stress, there have been some studies evaluating the utility of acupuncture in post-traumatic stress disorder, but again these results are limited and the long-term benefits come with limited confidence.

Insomnia is a sleep disorder characterized by trouble sleeping. It can occur independently or as a result of other medical conditions including stress, pain, or even heart failure. Typically it is managed by determining the precipitating cause and addressing it, but there are also many pharmacological approaches that can be taken. This route is usually less preferable when compared to non-medication based interventions including encouraging sleep hygiene, relaxation therapy and cognitive behavioral therapy. A systematic review and meta-analysis examining the effects of acupuncture on insomnia revealed statistically significant results in favor of benefits from acupuncture. It was found to be more effective than 
pharmacotherapy. 32

Tendonitis is defined as inflammation of a tendon. The symptomatology associated with this can range from aches and pains along with joint stiffness, to whole joint burning. Conservative treatment is typically first-line and involves the use of NSAIDs, rest and gradual return to prior activities. A single-blind, randomized, controlled trial on patients with shoulder pain and the diagnosis of osteoarthritis or rotator cuff tendonitis found that acupuncture may be effective for treating shoulder pain, and thus addressing the tendonitis. 33

Spinal stenosis can cause a number of uncomfortable symptoms such as numbness and weakness in the extremities, but it can also cause a number of serious issues such as incontinence. There are numerous causes of it and a number of treatment options as well. Non-surgical avenues are typically explored first and tend to have good outcomes. Acupuncture has been used to attempt and quell some of the symptoms associated with this condition. A systematic review and meta-analysis of 6 databases revealed that there was no conclusive evidence on the effectiveness and safety of acupuncture for lumbar spinal stenosis. ${ }^{34}$ Further research is needed in regards to this.

Post-surgical pain is a common symptom amongst patients who have undergone some sort of operative procedure. Surprisingly, some studies have shown that less than half of those individuals report adequate pain relief. Auricular acupuncture, peri-operatively, has increased in use with the goal of reducing post-operative pain. A recent systematic review found that patients treated with acupuncture or related techniques had less pain and used less opioid analgesics on Day 1 after surgery. Further research needs to be done in this regard, but the preliminary studies and findings are promising. 35

Osteoarthritis (OA) is one of the most common forms of joint disease that results in increased pain, loss of mobility and functional independence. The disease process involves destruction of underlying articular cartilage, affecting one in every five members of the aging population. ${ }^{36}$

Commonly affected joints include the fingers, neck, knee and hip, with increased risk for those who engage in activities involving high levels of joint stress. Current treatments commonly involve exercise, reducing joint stress and medications as NSAIDs and Acetaminophen.

With regard to OA of the knee, studies have shown that acupuncture in addition to pharmacological therapy is superior to pharmacological therapy alone for reducing pain, improving functionality and quality of life. ${ }^{37}$ Another meta-analysis also shows that proper acupuncture is more effective than sham 
treatment for chronic knee pain. ${ }^{38}$ Another study exploring the application of acupuncture for OA of the hip found drastic improvement in pain relief and reduced analgesic drug intake that lasted at least two months, when compared to traditional therapy. ${ }^{39}$ These findings necessitate additional studies to explore the benefits of acupuncture in osteoarthritic disease processes.

\section{CONCLUSION}

Acupuncture is a form of medicine that has been applied for centuries in the Eastern world. It is being used as part of a holistic approach to the treatment of pain syndromes in conjunction with diet, massage, and herbal therapy. As discussed above, it is a relatively new modality being used in the Western world either as a direct mechanism or an adjunct for pain relief. The information provided should help clinicians gain insight into this form of traditional Eastern medicine, the role of Meridians, and its proposed mechanism of action for pain relief.

While studies have suggested that the release of endogenous opioids provides the main analgesic effects of acupuncture, ${ }^{40}$ there are still questions surrounding its efficacy in clinical application, proper patient selection, and specific chronic pain syndromes that may benefit from use. It is readily apparent that there is a lack of consensus with regards to creating guidelines for conventional care and thus further research should be conducted regarding the efficacy of stand-alone acupuncture therapy or in conjunction with conventional therapy.

\section{REFERENCES}

1. Dharmananda S, Dorr C. Traditional View. An Introduction to Acupuncture and How It Works. http://www.itmonline.org/arts/acuintro.htm. Published 1996.

2. Acupuncture. Encyclopædia Britannica. https://www.britannica.com/science/acupuncture. Published March 22, 2018.

3. White A, Ernst E. A brief history of acupuncture. Rheumatology. 2004;43(5):662-665.

4. Lu Dominic P., Lu Gabriel P.. An Historical Review and Perspective on the Impact of Acupuncture on U.S. Medicine and Society. Medical Acupuncture. 2013;25(5):311-316.

5. Hopwood V.Acupuncture in physiotherapy: Key concepts and evidence-based practice. Philadelphia: Elsevier Limited; 2004.

6. Wilkinson Jonathan, Faleiro Richard. Acupuncture in pain management. Continuing Education in Anaesthesia Critical Care \& Pain. 2007;7(4):135-138. 
7. Longhurst John C.. Defining Meridians: A Modern Basis of Understanding. Journal of Acupuncture and Meridian Studies. 2010;3(2):67-74.

8. Carlsson C. Acupuncture mechanisms for clinically relevant long-term effects: reconsideration and a hypothesis. Acupunct Med. 2002;20:82-99.

9. He L, Zhou M, Zhou D, Wu B, Li N, Kong S Y.Acupuncture for Bell's palsy.. Cochrane Database Syst Rev. 2007;:2914-2914.

10. Neurobiological Mechanisms of Acupuncture for Some Common Illnesses: A Clinician's Perspective. Cheng, Kwokming James. Journal of Acupuncture and Meridian Studies. ;7(3):105-114.

11. Bai L, Yan H, Li L, Qin W, Chen P, Liu P. Neural specificity of acupuncture stimulation at pericardium 6: evidence from an FMRI study. J Magn Reson Imaging. 2010;31:71-77.

12. Rittner H L, Brack A, Machelska H, et al. Opioid peptide-expressing leukocytes: Identification, recruitment, and simultaneously increasing inhibition of inflammatory pain. Anesthesiology. 2001;95:500-508.

13. Cabot P J, Carter L, Gaiddon C, et al. Immune cell-derived beta-endorphin. Production, release, and control of inflammatory pain in rats.. Journal of Clinical Investigation. 1997;100(1):142-148.

14. Anand Praveen, Whiteside Garth, Fowler Christopher J., Hohmann Andrea G.. Targeting CB2 receptors and the endocannabinoid system for the treatment of pain. Brain Research Reviews. 2009;60(1):255-266.

15. Su T.F., Zhao Y.Q., Zhang L. H., et al. Electroacupuncture reduces the expression of proinflammatory cytokines in inflamed skin tissues through activation of cannabinoid CB2 receptors. European Journal of Pain. 2012;16(5):624-635.

16. Sekido Reina, Ishimaru Keisou, Sakita Masakazu. Corticotropin-Releasing Factor and Interleukin-1 $\beta$ are Involved in the Electroacupuncture-Induced Analgesic Effect on Inflammatory Pain Elicited by Carrageenan. The American Journal of Chinese Medicine. 2004;32(02):269-279.

17. Zhao Z Q. Neural mechanism underlying acupuncture analgesia. Prog Neurobiol. 2008;85:355-75.

18. Qu S S, Huang Y,Zhang Z J, et al. A 6-week randomized controlled trial with 4-week follow-up of acupuncture combined with paroxetine in patients with major depressive disorder. J Psychiatr Res. 
2013;47:726-758.

19. Shan Sun, Qi-Liang Mao-Ying, Hong Cao, et al. Is functional state of spinal microglia involved in the anti-allodynic and anti-hyperalgesic effects of electroacupuncture in rat model of monoarthritis?. Neurobiology of Disease. 2007;26(3):558-568.

20. BH., Dubois D., Gendron L.. Activation of Spinal - and -Opioid Receptors Potently Inhibits Substance P Release Induced by Peripheral Noxious Stimuli. Journal of Neuroscience. 2011;31(37):13068-13077.

21. Choi Doo C., Lee Jee Y., Lim Eun J., Baik Hyung H., Oh Tae H., Yune Tae Y.. Inhibition of ROS-induced p38MAPK and ERK activation in microglia by acupuncture relieves neuropathic pain after spinal cord injury in rats. Experimental Neurology. 2012;236(2):268-282.

22. Zhang Ruixin. Mechanisms of Acupuncture-Electroacupuncture on Persistent Pain. Anesthesiology. 2014;120:482-503.

23. Chu W C, Wu J C, Yew D T, et al. Does acupuncture therapy alter activation of neural pathwayfor pain perception in irritable bowel syndrome?: A comparative study of true and sham acupuncture using functional magnetic resonance imaging. J Neurogastroenterol Motil.2012;18:305-321.

24. Lin Katerina, Tung Cynthia. The Regulation of the Practice of Acupuncture by Physicians in the United States. Medical Acupuncture. 2017;29(3):121-127.

25. Vickers Andrew J, Rees Rebecca W, Zollman Catherine E, et al. Acupuncture for chronic headache in primary care: large, pragmatic, randomised trial. BMJ. 2004;328:744-744.

26. Haake M, Müller H, Schade-Brittinger C. Acupuncture Trials (Gerac) For Chronic Low Back PainRandomized, Multicenter, Blinded, Parallel-Group Trial With 3 Groups. Arch Intern Med. 2007;167(17):1892-1898.

27. Lee J H, Choi T Y, Lee M S, Lee H, Shin B C, Lee H. Acupuncture for Acute Low Back Pain. The Clinical Journal of Pain. 2013;29(2):172-185.

28. Deare John C, Zheng Zhen, Xue Charlie CL, et al. Acupuncture for treating fibromyalgia. Cochrane Database of Systematic Reviews. 2013;.

29. Ji Mei, Wang Xiaoxia, Chen Meijuan, Shen Yan, Zhang Xu, Yang Jin. The Efficacy of Acupuncture 
for the Treatment of Sciatica: A Systematic Review and Meta-Analysis. Evidence-Based Complementary and Alternative Medicine. 2015;2015:1-12.

30. Wang R, Li X, Zhou S. Manual acupuncture for myofascial pain syndrome: a systematic review and meta-analysis Acupuncture in. Medicine. 2017;35:241-250.

31. Pavão Tiago S., Vianna Priscila, Pillat Micheli M., Machado Amanda B., Bauer Moisés E.. Acupuncture is effective to attenuate stress and stimulate lymphocyte proliferation in the elderly. Neuroscience Letters. 2010;484(1):47-50.

32. Shergis Johannah Linda, Ni Xiaojia, Jackson Melinda L., et al. A systematic review of acupuncture for sleep quality in people with insomnia. Complementary Therapies in Medicine. 2016;26:11-20.

33. Lathia Amanda Tiffany, Jung S. M., Chen Lan X.. Efficacy of Acupuncture as a Treatment for Chronic Shoulder Pain. The Journal of Alternative and Complementary Medicine. 2009;15(6):613-618.

34. Acupuncture for Lumbar Spinal Stenosis: A Systematic Review and Meta-Analysis. Complementary Therapies in Medicine. 2013;.

35. Wu M S, Chen K H, Chen I F. The Efficacy of Acupuncture in Post-Operative Pain Management: A Systematic Review and Meta-Analysis. PLoS One. 2009;11(3).

36. Lawrence R C, Hochberg M C, Kelsy J L, Mcduffie F C, Medsger T A. Estimates of the prevalence of selected arthritic and musculoskeletal diseases in the United States. J Rheumatol. 1989;16(4):427-468.

37. Vas Jorge, Méndez Camila, Perea-Milla Emilio, et al. Acupuncture as a complementary therapy to the pharmacological treatment of osteoarthritis of the knee: randomised controlled trial. BMJ. 2004;329(7476):1216-1216.

38. White A., Foster N. E., Cummings M., Barlas P.. Acupuncture treatment for chronic knee pain: a systematic review. Rheumatology. 2007;46(3):384-390.

39. Haslam R. A comparison of acupuncture with advice and exercises on the symptomatic treatment of osteoarthritis of the hip - a randomised controlled trial. Acupuncture in Medicine. 2001;19:19-26.

40. Melzack R., Wall P. D.. Pain Mechanisms: A New Theory. Science. 1965;150(3699):971-978. 